Journal for ImmunoTherapy of Cancer

\title{
Anticancer immunity induced by a synthetic tumor-targeted CD137 agonist
}

\author{
Punit Upadhyaya, ${ }^{1}$ Johanna Lahdenranta, ${ }^{1}$ Kristen Hurov, ${ }^{1}$ Sailaja Battula, ${ }^{1}$ \\ Rachel Dods, ${ }^{2}$ Eric Haines, ${ }^{1}$ Marianna Kleyman, ${ }^{1}$ Julia Kristensson, ${ }^{2}$ \\ Jessica Kublin, ${ }^{1}$ Rachid Lani, ${ }^{2}$ Jun Ma, ${ }^{1}$ Gemma Mudd, ${ }^{2}$ Elizabeth Repash, \\ Katerine Van Rietschoten, ${ }^{2}$ Tom Stephen, ${ }^{1}$ Fanglei You, ${ }^{1}$ Helen Harrison, ${ }^{2}$ \\ Liuhong Chen, ${ }^{2}$ Kevin McDonnell, ${ }^{1}$ Philip Brandish, ${ }^{1}$ Nicholas Keen
}

To cite: Upadhyaya $P$, Lahdenranta J, Hurov K, et al. Anticancer immunity induced by a synthetic tumor-targeted CD137 agonist. Journal for ImmunoTherapy of Cancer 2021;9:e001762. doi:10.1136/ jitc-2020-001762

- Additional material is published online only. To view, please visit the journal online (http://dx.doi.org/10.1136/jitc2020-001762).

$\mathrm{PU}, \mathrm{JL}$ and $\mathrm{KH}$ contributed equally.

$\mathrm{PU}, \mathrm{JL}$ and $\mathrm{KH}$ are joint first authors.

Accepted 15 December 2020

Check for updates

(c) Author(s) (or their employer(s)) 2021. Re-use permitted under CC BY-NC. No commercial re-use. See rights and permissions. Published by BMJ.

${ }^{1}$ Bicycle Therapeutics, Lexington, Massachusetts, USA

${ }^{2}$ Bicycle Therapeutics, Babraham Research Campus, UK

Correspondence to

Dr Nicholas Keen;

nicholas.keen@bicycletx.com

\section{ABSTRACT}

Background In contrast to immune checkpoint inhibitors, the use of antibodies as agonists of immune costimulatory receptors as cancer therapeutics has largely failed. We sought to address this problem using a new class of modular synthetic drugs, termed tumor-targeted immune cell agonists (TICAs), based on constrained bicyclic peptides (Bicycles).

Methods Phage libraries displaying Bicycles were panned for binders against tumor necrosis factor (TNF) superfamily receptors CD137 and 0X40, and tumor antigens EphA2, Nectin-4 and programmed death ligand 1. The CD137 and OX40 Bicycles were chemically conjugated to tumor antigen Bicycles with different linkers and stoichiometric ratios of binders to obtain a library of low molecular weight TICAs ( $\mathrm{MW}<8 \mathrm{kDa}$ ). The TICAs were evaluated in a suite of in vitro and in vivo assays to characterize their pharmacology and mechanism of action.

Results Linking Bicycles against costimulatory receptors (e.g., CD137) to Bicycles against tumor antigens (e.g., EphA2) created potent agonists that activated the receptors selectively in the presence of tumor cells expressing these antigens. An EphA2/CD137 TICA (BCY12491) efficiently costimulated human peripheral blood mononuclear cells in vitro in the presence of EphA2 expressing tumor cell lines as measured by the increased secretion of interferon $\gamma$ and interleukin-2. Treatment of C57/BI6 mice transgenic for the human CD137 extracellular domain (huCD137) bearing EphA2-expressing MC38 tumors with BCY12491 resulted in the infiltration of CD8+ T cells, elimination of tumors and generation of immunological memory. BCY12491 was cleared quickly from the circulation (plasma $t_{1 / 2}$ in mice of 1-2 hr), yet intermittent dosing proved effective.

Conclusion Tumor target-dependent CD137 agonism using a novel chemical approach (TICAs) afforded elimination of tumors with only intermittent dosing suggesting potential for a wide therapeutic index in humans. This work unlocks a new path to effective cancer immunotherapy via agonism of TNF superfamily receptors.

\section{BACKGROUND}

The tumor necrosis factor (TNF) receptor superfamily members, including CD137/4-1BB, OX40 and CD40, are important regulators of immune cell function. ${ }^{1}$ Agonistic antibodies have been investigated extensively as cancer immunotherapies with disappointing clinical outcomes despite a strong mechanistic rationale for utility and preclinical proof of concept. ${ }^{2-4}$ Translation of efficacy from preclinical mouse models to human cancer is, in general, notoriously poor. ${ }^{5}$ However, other factors related to the modality, including the duration and location of action of the drugs that have been tested in this class, suggested to us that a more fit-for-purpose pharmacological approach may yield meaningful clinical benefit to patients with few treatment options. Specifically, in the case of CD137, those factors include (1) slow penetration of antibodies into tumors leading to peripheral rather than intratumoral agonism, ${ }^{6}$ (2) sustained agonism from continuous exposure resulting in overstimulation and activationinduced cell death of lymphocytes ${ }^{78}$ and (3) Fc receptor interactions that drive the doselimiting toxicity observed with urelumab. ${ }^{9} 10$ Constrained bicyclic peptides or Bicycles are ideally positioned to address these challenges in a way not practically feasible with traditional antibodies ${ }^{23911}$ or bispecific biological agents. ${ }^{12-14}$ Bicycles offer small highly modular building blocks, ${ }^{15} 16$ rapid tissue distribution and penetration, ${ }^{17}$ rapid clearance compared with biologics ${ }^{16} 18$ and drug-like characteristics. $^{18} 19$

\section{METHODS}

\section{Cell lines and reagents}

HT-1376， NCI-H292， PC-3， RKO， A549, LNCaP, HT-29, HT-1080, CT26 and 4T1 cells were obtained from ATCC. MC38 cells were obtained from the National Cancer Institute (L-159-2018/1).

4T1 cells were engineered to express mouse Nectin-4 (NM_027893.3) using CRISPR/ 
Cas9 gene editing. In brief, a targeting vector carrying the mouse Nectin- 4 coding sequence and homology arms recognizing the Rosa26 locus was transiently transfected into 4T1 cells along with plasmids expressing the appropriate sgRNAs. Single cell clones were isolated, and mouse Nectin- 4 levels were evaluated by flow cytometry.

Nectin-4 overexpressing MC38 and CT26 cell lines were generated by lentiviral infection using pLenti6.3CMV-MCS vector (Invitrogen) with Nectin4 CDS region (NM_027893.3) at MCS (AscI and PmeI). Virus was prepared by transfecting 293FT cells (Invitrogen) with psPAX, VSV-G and Nectin-4 (or NT) vectors. CT26 or MC38 cells were transduced by Nectin- 4 or NT lentivirus and selected with Blasticidin. Several individual clones were screened by flow cytometry (anti-Nectin-4, AF2659, R\&D Systems) to select clones with appropriate level of Nectin-4 expression. Clones CT26\#7 and MC38\#13 were selected for further studies. Nectin-4 overexpression in CT26\#7 and MC38\#13 tumor tissues was verified by flow cytometry from single cell suspensions prepared from tumors grown in syngeneic Balb/c (CT26\#7) or C57Bl/6 (MC38\#13) mice.

Human peripheral blood mononuclear cells (PBMCs) from healthy volunteer donors were isolated from buffy coats (Oklahoma Blood Institute, Oklahoma City, Oklahoma, USA) by ficoll centrifugation gradient (GE Healthcare Ficoll-Paque PLUS Media), followed by red blood cell lysis with ACK lysing buffer (Gibco).

Anti-CD137 mAbs: Urelumab was prepared by Biocytogen (Beijing, China) using ExpiCHO expression system via transient transfection using sequence from published patent (US8137667B2) and purified by protein A affinity chromatography or purchased from Creative Biolabs (TAB-179). Utomilumab was prepared using HEK293 expression system via transient transfection using heavy chain (LC) and light chain (HC) regions from published patent (US20120237498A1) and purified using protein $\mathrm{G}$ affinity chromatography.

Recombinant proteins: The following proteins were purchased for use in SPR experiments or in vitro reporter cell assays. Human proteins: CD137 (92204B, R\&D Systems), CD137L (2295-4L, R\&D Systems), OX40-Fc (OXO-H5255, Acro Biosystems), OX40 (OX0H5224, Acro Biosystems), OX40L (OXL-H52Q8, Acro Biosystems), CD40 (CD40-H5228, Acro Biosystems), programmed death ligand 1 (PD-L1) (9049-B7, R\&D Systems), EphA1-Fc (15789-H02H, Sino Biologics), EphA3-Fc (6444-A3, R\&D Systems), EphA4-Fc (11314H03H, Sino Biologics), EphA5 (3036-A5, R\&D Systems), EphA6-Fc (5606-A6, R\&D Systems), EphB4-Fc (10235H02H, Sino Biologics), Nectin-1 (2880-N1, R\&D Systems), Nectin-2 (2229-N2, R\&D Systems), Nectin-3 (3064-N3, R\&D Systems), Nectin-like-1 (3678-S4-050, R\&D Systems), Nectin-like-2 (3519-S4-050, R\&D Systems), Nectin-like-3 (4290-S4-050, R\&D Systems), Nectin-like-4 (4164-S4, R\&D Systems) and Nectin-like-5 (2530-CD-050, R\&D Systems). Mouse proteins: CD137 (41B-M52H7, Acro Biosystems), EphA2 (50586-M08H, Sino Biological) Nectin-4
(3116-N4, R\&D Systems) and PD-L1 (9048-B7-100, R\&D Systems).

The following proteins were expressed for use in SPR experiments. Human EphA2: The ecto domain of human EphA2 (Lys27-Asn529) was cloned into pEXPR-IBA44, between the 5' NheI and 3' BsaI sites. Protein was expressed in HEK293 cells. It was purified by IMAC and gel filtration chromatography. Human Nectin-4 was prepared at Charles River Labs. Human Nectin-4 (residues Gly32-Ser349; NCBI RefSeq: NP_112178.2) with a gp67 signal sequence and C-terminal FLAG tag was cloned into pFastbac-1 and baculovirus made using standard Bac-to-Bac protocols (Life Technologies). Sf21 cells at $1 \times 10^{6} / \mathrm{mL}$ in Excell-420 medium (Sigma) at $27{ }^{\circ} \mathrm{C}$ were infected at a MOI of 2 with a P1 virus stock and the supernatant harvested at $72 \mathrm{hr}$. The supernatant was batch bound for $1 \mathrm{hr}$ at $4{ }^{\circ} \mathrm{C}$ with Anti-FLAG M2 affinity agarose resin (Sigma) washed in PBS and the resin subsequently transferred to a column and washed extensively with PBS. The protein was eluted with $100 \mu \mathrm{g} / \mathrm{mL}$ FLAG peptide. The eluted protein was concentrated to $2 \mathrm{~mL}$ and loaded onto an S-200 superdex column (GE Healthcare) in PBS at $1 \mathrm{~mL} / \mathrm{min}$. Two $\mathrm{mL}$ fractions were collected and the fractions containing Nectin-4 protein were concentrated to $16 \mathrm{mg} / \mathrm{mL}$.

Methods for receptor quantitation, multimer binding to CD8+ T cells, phage display and affinity maturation, crystallography and structure determination, fluorescent polarization competition assays, surface plasmon resonance binding assays, synthesis and purification of the molecules can be found in the (online supplemental materials).

\section{Reporter assays}

CD137 and OX40 Bioassay kits (Promega) were carried out according to the manufacturer's protocol. Briefly, a Jurkat $\mathrm{T}$ cell line engineered to express human CD137 or OX40 and a luciferase reporter driven by a response element that can respond to CD137 or OX40 ligand/ agonist antibody stimulation were cultured at $37{ }^{\circ} \mathrm{C}, 5 \%$ $\mathrm{CO}_{2}$ for $6 \mathrm{hr}$ with or without test article. After $6 \mathrm{hr}$, BioGlo Luciferase reagent was added to each well. Luminescence was measured on a BMG CLARIOStar microplate reader. Fold induction was calculated as relative light unit (RLU) divided by RLU of background (no test article) wells. For reporter assays done in co-culture, 10,000 cells of the indicated tumor cell line were added to each well of the 96 well plate at the start of culture.

\section{Cytokine release assays and cytokine quantification}

Frozen PBMCs from healthy human donors were thawed and washed one time in room temperature PBS with benzonase, and then resuspended in RPMI supplemented with $10 \%$ heat inactivated fetal bovine serum, 1x penicillin/streptomycin, $10 \mathrm{mM}$ HEPES, and $2 \mathrm{mM}$ L-glutamine (herein referred to as R10 medium). Mouse (MC38 or MC38\#13) or human (HT-29, PC3, HT-1080, or HT-1376) tumor cells were cultured in the appropriate 
media. $100 \mu \mathrm{L}$ of PBMCs $\left(1 \times 10^{6} / \mathrm{mL}\right)$ and $100 \mu \mathrm{L}$ of tumor cells $\left(1 \times 10^{5} / \mathrm{mL}\right)$ (effector:target cell ratio 10:1) were plated in each well of a 96 well flat bottom plate for the co-culture assay. $100 \mathrm{ng} / \mathrm{ml}$ of soluble anti-CD3 $\mathrm{mAb}$ (clone OKT3; Biolegend) was added to the culture on day 0 to stimulate human PBMCs. Test, control compounds, or vehicle controls were diluted in R10 media and $50 \mu \mathrm{L}$ was added to respective wells to bring the final volume per well to $250 \mu \mathrm{L}$. Plates were covered with a breathable film and incubated in a humidified chamber at 37 ${ }^{\circ} \mathrm{C}$ with $5 \% \mathrm{CO}_{2}$ for 2 days. Supernatants were collected 24 and $48 \mathrm{hr}$ after stimulation, and human interleukin-2 (IL-2) and interferon gamma (IFNY) was detected by Luminex. Briefly, the standards and samples were added to a black 96-well plate. Microparticle cocktail (provided in Luminex kit, R\&D Systems) was added and shaken for $2 \mathrm{hr}$ at room temperature. The plate was washed 3 times using magnetic holder. Biotin cocktail was then added to the plate and shaken for $1 \mathrm{hr}$ at RT. The plate was washed 3 times using magnetic holder. Streptavidin cocktail was added to the plate and shaken for $30 \mathrm{~min}$ at RT. The plates were washed 3 times using magnetic holder, resuspended in $100 \mu \mathrm{L}$ of wash buffer, shaken for $2 \mathrm{~min}$ at RT, and read using the Luminex 2000. Raw data were analyzed using built-in Luminex software to generate standard curves and interpolate protein concentrations, all other data analyses and graphing were performed using Excel and Prism software.

\section{Pharmacokinetics of BCY10572 (IV) and BCY12491 (IP) in CD-1 mouse}

Male CD- 1 mice were dosed with $5 \mathrm{mg} / \mathrm{kg}$ of BCY10572 via tail vein injection or $15 \mathrm{mg} / \mathrm{kg}$ BCY12491 intraperitoneally formulated in $25 \mathrm{mM}$ histidine $\mathrm{HCl}, 10 \%$ sucrose $\mathrm{pH}$ 7. Serial bleeding was performed via submandibular or saphenous vein at each time point. All blood samples were immediately transferred into prechilled microcentrifuge tubes containing K2-EDTA $(0.5 \mathrm{M})$ as anticoagulant and placed on wet ice. Blood samples were immediately processed for plasma by centrifugation at approximately $4{ }^{\circ} \mathrm{C}, 3000 \mathrm{~g}$. The precipitant including internal standard was immediately added into the plasma, mixed well and centrifuged at $12,000 \mathrm{rpm}, 4^{\circ} \mathrm{C}$ for $10 \mathrm{~min}$. The supernatant was transferred into prelabeled polypropylene microcentrifuge tubes, and then quick-frozen over dry ice. The samples were stored at $70{ }^{\circ} \mathrm{C}$ or below as needed until analysis. The samples were analyzed by injecting $7.5 \mu \mathrm{L}$ of the supernatant into Orbitrap Q Exactive in positive ion mode for BCY12491 and Triple Quad 6500 Plus for BCY10572. Plasma concentration versus time data were analyzed by non-compartmental approaches using the Phoenix WinNonlin V.6.3 software program. $\mathrm{C}_{0}, \mathrm{Cl}, \mathrm{Vd}_{\mathrm{ss}}$, $\mathrm{T}_{1 / 2}, \mathrm{AUC}_{(0-\text {-ast })}, \mathrm{AUC}_{(0-\text { inf })}, \mathrm{MRT}_{(0 \text {-last })}, \mathrm{MRT}_{(0 \text {-inf) }}$ and graphs of plasma concentration versus time profile were reported.

\section{Syngeneic tumor models}

Female C57BL/6J-hCD137 mice (B-hTNFRSF9(CD137) mice aged 6-8 weeks; Biocytogen, Beijing, China) were implanted subcutaneously with $1 \times 10^{6}$ MC38 cells. Mice were randomized into treatment groups when average tumor volumes reached $76 \mathrm{~mm}^{3}$ and were treated with vehicle (25 mM histidine, 10\% sucrose, $\mathrm{pH} 7$; QDx19, ip) or BCY12491 at $5 \mathrm{mg} / \mathrm{kg}$ or $15 \mathrm{mg} / \mathrm{kg}$ (QDx21, ip), or $5 \mathrm{mg} / \mathrm{kg}$ or $15 \mathrm{mg} / \mathrm{kg}$ (Q3Dx8, ip). Tumor growth was monitored by caliper measurements. Tumor volume is expressed in $\mathrm{mm}^{3}$ using the formula: $\mathrm{V}=0.5 \mathrm{a} \times \mathrm{b}^{2}$ where $\mathrm{a}$ and $\mathrm{b}$ are the long and short diameters of the tumor, respectively. Mice cured by the BCY12491 treatment were re-inoculated subcutaneously to bilateral flank with $1 \times 10^{6}$ MC38 cells 81 days after the treatment initiation and tumor growth was monitored until day 31 post rechallenge. As a control, 5 naive age-matched C57BL/6J-hCD137 mice were inoculated in parallel and tumor growth was monitored as described.

For testing the multimeric CD137 Bicycle agonist BCY8947, MC38 tumor bearing C57BL/6J-hCD137 mice were randomized to receive vehicle, $30 \mathrm{mg} / \mathrm{kg}$ BCY8947 IP QD or $3 \mathrm{mg} / \mathrm{kg}$ anti-CD137 agonist antibody (urelumab analogue, Biocytogen) intraperitoneal two times a week (BIW) when average tumor volume reached $110 \mathrm{~mm}^{3}$. Tumor growth was monitored as described above. Single cell suspensions prepared from tumors were analyzed for $\mathrm{T}$ cells $(\mathrm{CD} 3+\mathrm{CD} 45+)$ and $\mathrm{CD} 8+\mathrm{T}$ cells $(\mathrm{CD} 8+\mathrm{CD} 3+\mathrm{CD} 45+)$ by flow cytometry. CD45 antibody (cat\#103138), CD3 antibody (cat\# 100210) and CD8 antibody (cat\#100759) were from Biolegend.

For the transcriptional and immunohistochemical (IHC) analyses, mice (as above) were implanted subcutaneously with 1 × $10^{6}$ MC38 cells. Mice were randomized into treatment groups when average tumor volumes reached around $240 \mathrm{~mm}^{3}$ and were treated intravenously with vehicle (25 mM histidine, $10 \%$ sucrose, $\mathrm{pH} 7), 15$ $\mathrm{mg} / \mathrm{kg}$ BCY12491, $15 \mathrm{mg} / \mathrm{kg}$ BCY13626 (non-binding EphA2/CD137 TICA control) or intraperitoneally with $2 \mathrm{mg} / \mathrm{kg}$ anti-CD137 antibody urelumab (determined to be maximally efficacious dose by 2 weeks of treatment, data not shown). Treatments were given Q3D for 3 doses and tumor tissues were harvested $1 \mathrm{hr}$ after the last dose. Tumors were categorized as ' $\mathrm{G}$; Growing' if tumor volume on day 6 had increased more than $10 \%$ from day 3 , 'R; Regressing' if tumor volume on day 6 had decreased from day 3 and 'S; Stable' if tumor volume on day 6 had increased less than $10 \%$ from day 3. Part of the tumor tissue was used for RNA isolation for transcriptional analysis and a part of the tumor tissue was used for formalinfixed paraffin embedded (FFPE) sample preparation for IHC analysis. RNA was isolated from tumor tissues using RNAeasy kit (Qiagen) and transcriptional analysis was performed using nCounter mouse PanCancer IO 360 panel (Nanostring) from $100 \mathrm{ng} \mathrm{RNA} /$ tumor. Data were analyzed using the nSolver analysis software with advanced analysis probe set ns_mm_io_360_v1.0 (Nanostring). CD8+ tumor infiltrating cells were stained in FFPE tissue sections using anti-mouse CD8 antibody (Abcam, \# ab217344) and Ventana Discovery OmniMap antirabbit-HRP kit (Ventana \#760 4310). 
For the immune cell depletion experiment, mice (as above) were implanted subcutaneously with $1 \times 10^{6}$ MC38\#13 (clone of MC38 that has been engineered to overexpress Nectin-4) cells. Three days after cell implantation mice received an intraperitoneal injection of vehicle (PBS), $100 \mu \mathrm{g}$ of depleting anti-CD8 (rat IgG2b, clone 2.42) or anti-NK (mouse IgG2a, clone PK136) antibodies (or their combination) or the corresponding isotype control antibodies (rat IgG2b isotype control or mouse IgG2a isotype control). Mice received additional doses of depletion antibodies (or isotype controls) 5 and 10 days after the first dose of antibodies. Cell depletion was verified by flow cytometry 4 and 12 days after the first dose of depletion antibody (online supplemental figure S6). When tumor volumes reached $111 \mathrm{~mm}^{3}$ on average (5 days after the first dose of depletion antibodies), mice started receiving vehicle or BCY12491 intravenously at $15 \mathrm{mg} / \mathrm{kg}$ BIW. Mice received a total of 4 doses of BCY12491. Tumor growth was monitored as described above. All depletion antibodies were InVivoPlus antibodies from BioXcell (Lebanon, New Hampshire, USA).

All the procedures related to animal handling, care and treatment in the studies were performed according to the guidelines approved by the Institutional Animal Care and Use Committee (IACUC) of WuXi AppTec (Beijing, China), following the guidance of the Association for Assessment and Accreditation of Laboratory Animal Care.

\section{Verification of in vivo immune cell depletion by FACS}

CD8 and NK cell depletion from circulation was verified by flow cytometry 5 days after the first dose of $\mathrm{T}$ cell or NK cell depleting antibodies (3 animals per treatment cohort). $150 \mu \mathrm{L}$ of anticoagulated (K2-EDTA) blood (submandibular bleed) was lysed (1:19) with RBC lysis solution (1x BD PharmLyse, BD Biosciences) and cells were washed with DPBS. Cells were stained with the following panel: Live/Dead fixable violet cell stain (Invitrogen), CD45-AF700 (30-F11), CD3-APC-Cy7 (1452C11), CD4-BV510 (RM4-5), CD8-BUV395 (53-6.7), NK1.1-BV786 (PK136) and CD335-FITC (89A1.4). All antibodies are from BD Biosciences. Stained cells were analyzed by BD LSR Fortessa and the data was analyzed using FlowJo software (gating scheme in online supplemental figure S6C).

\section{RESULTS \\ Bicycle tool kit for TNF receptor agonism}

Bicycles are discovered via phage display of peptides (figure 1A,B). ${ }^{20}$ Displayed peptides $9-15$ amino acids in length are chemically cyclized on the bacteriophage surface and phage are selected based on binding to the target protein. The amino acid sequences of the peptide binders can be determined by sequencing of the phage DNA, and their binding affinities improved by further rounds of diversification and affinity selection. Finally, the peptides are chemically synthesized, and their properties

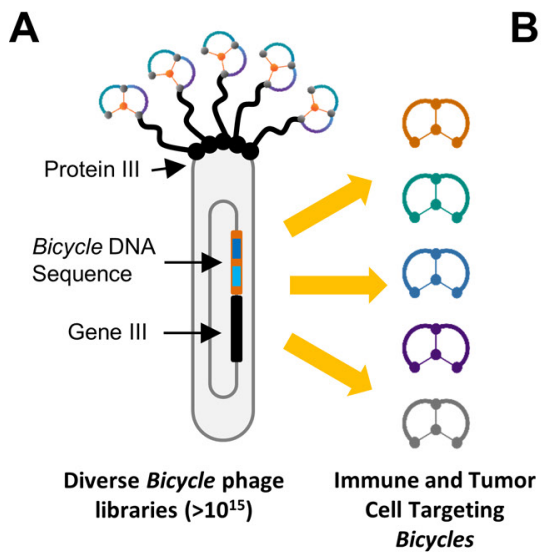

\begin{tabular}{lccc}
\hline $\begin{array}{l}\text { Target/ } \\
\text { Bicycle }\end{array}$ & $\begin{array}{c}\text { KD } \\
\text { human } \\
\text { (nM) }\end{array}$ & $\begin{array}{c}\text { KD } \\
\text { mouse } \\
\text { (nM) }\end{array}$ & $\begin{array}{c}\text { Specificity } \\
\text { Demonstrated }\end{array}$ \\
\hline $\begin{array}{l}\text { CD137 } \\
\text { BCY7965 }\end{array}$ & 4.4 & nb & $\checkmark$ \\
\hline $\begin{array}{l}\text { OX40 } \\
\text { BCY10549 }\end{array}$ & 637 & nd & nd \\
\hline $\begin{array}{l}\text { EphA2 } \\
\text { BCY6099 }\end{array}$ & 1.7 & 1.8 & $\checkmark$ \\
\hline $\begin{array}{l}\text { Nectin-4 } \\
\text { BCY8116 }\end{array}$ & 0.69 & 1.2 & $\checkmark$ \\
\hline $\begin{array}{l}\text { PD-L1 } \\
\text { BCY10467 }\end{array}$ & 4.3 & nb & nd \\
\hline
\end{tabular}
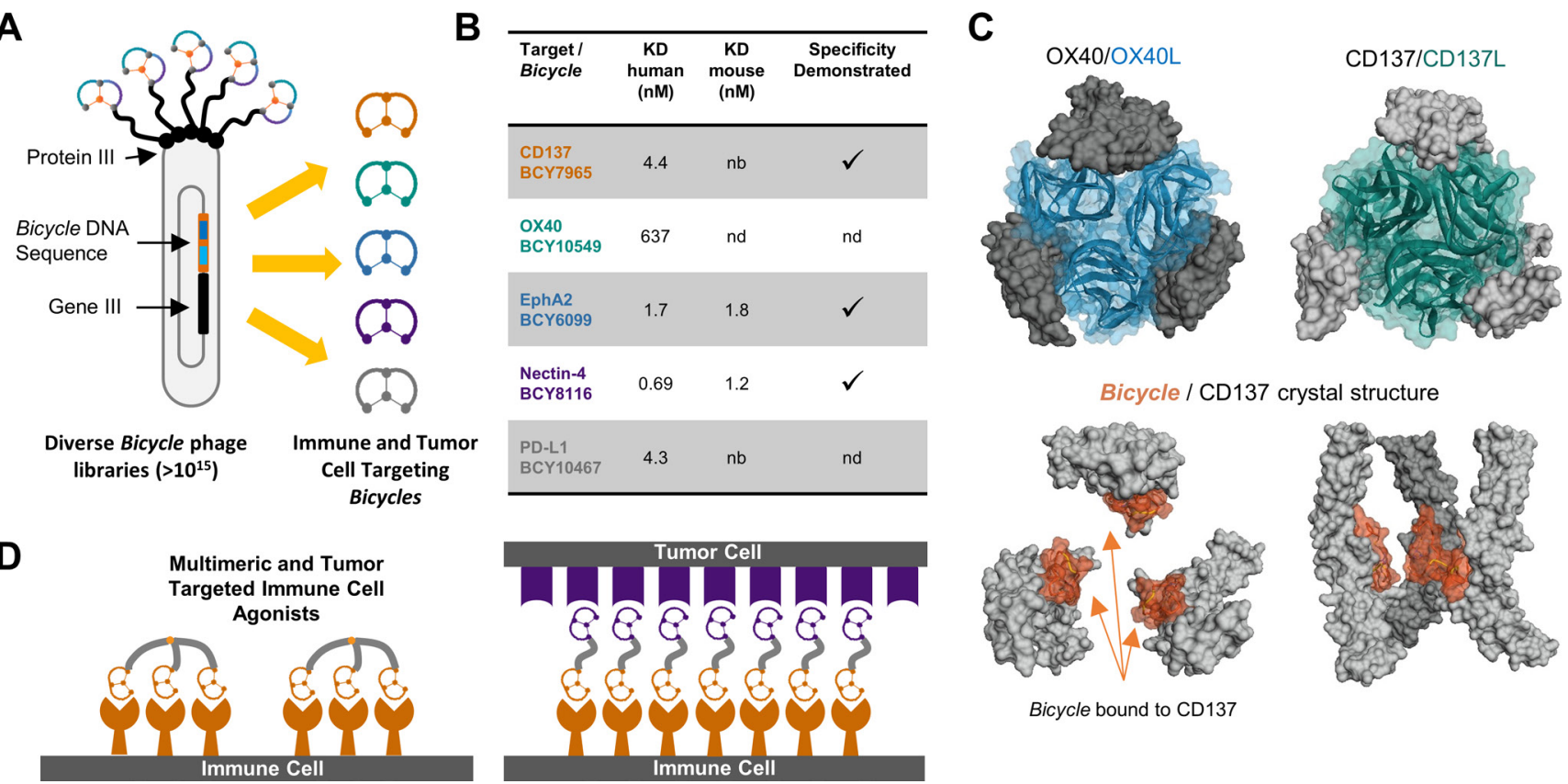

Bicycle bound to CD137

Figure 1 Generation of a Bicycle tool kit. (A) Schematic representation of the discovery of Bicycle binders using phage display with on-phage cyclization. (B) Biochemical properties of prototypical Bicycle binders. Potencies were determined by surface plasmon resonance (SPR). Specificity of EphA2, Nectin-4 and CD137 Bicycle binders were evaluated using SPR with other ephrin, Nectin-4 and TNF family receptors (OX40 and CD40), respectively ( $\mathrm{nb}=$ no binding, nd=not determined). (C) Comparison of three bound CD137 Bicycle (BCY10916, Protein data bank (PDB) accession number 6Y8K) complexes versus CD137/ CD137L (6MGP) and OX40/OX40L (2HEV) trimer complexes. (D) Illustration of the potential binding modes for multimeric Bicycle agonists (left) or tumor-targeted immune cell agonists (TICAs, right). TNF, tumor necrosis factor. 
A

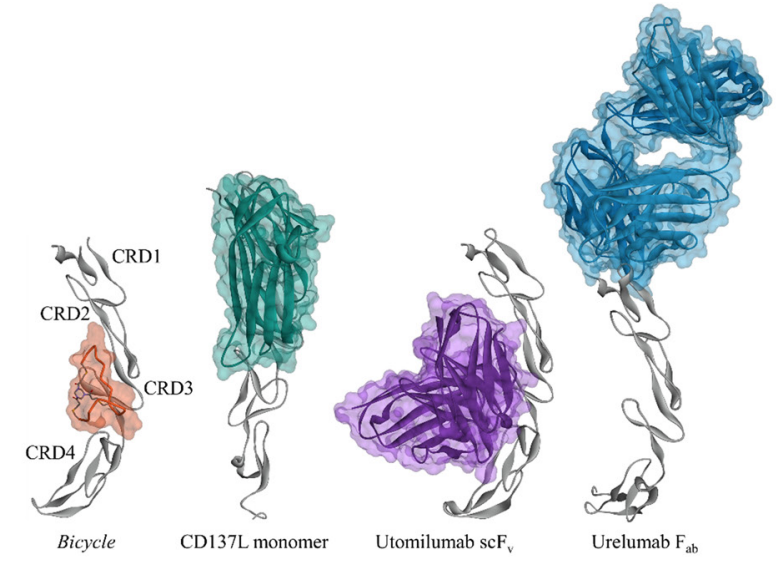

B

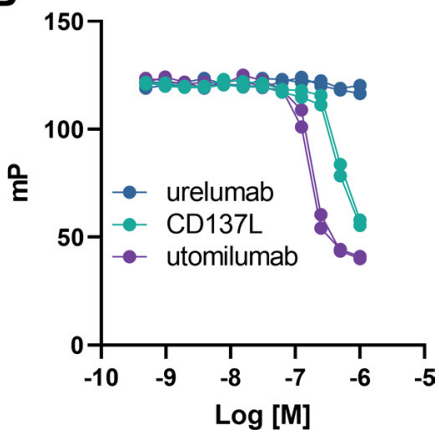

Figure 2 Modes of interaction and competitive binding analyses for CD137L, a CD137 Bicycle and anti-CD137 antibodies. (A) An analog of phage affinity matured CD137 Bicycle BCY592 (BCY10916, orange) binds to CD137 (gray) at CRD domains 2 and 3 (PDB accession 6Y8K). Comparison of the Bicycle CD137 binding site illustrates partial overlap with both the weakly agonistic antibody utomilumab (purple; scFv variant shown; PDB accession 6A3W) and CD137L (green; only monomer shown for clarity; PDB accession 6MGP). Urelumab (blue; Fab shown; PDB: 6MHR) binds distally to the $\mathrm{N}$-terminal CRD one region and does not overlap with the Bicycle binding site. (B) Fixed concentration of fluorescent analog of CD137 Bicycle BCY592 (BCY640) was incubated with $500 \mathrm{nM}$ of human CD137 and increasing concentrations of hCD137L, urelumab analog or utomilumab analog were added as competitors and fluorescent polarization was measured. The data represent values from two independent experiments. CRDs, cysteine-rich domains.

are further tuned as needed by the introduction of nonnatural amino acids. ${ }^{15}$

In this way, we created a 'tool kit' comprising potent and selective Bicycles that bind to two TNF receptors (TNFRs) (CD137, OX40) and to three well-known tumor cell-expressed antigens (EphA2, Nectin-4 and PD-L1) (figure 1B). This unique discovery process is exemplified by the discovery of CD137 binding Bicycles as follows: A prototype CD137 Bicycle binder (BCY586) was identified using phage display $\left(\mathrm{K}_{\mathrm{D}}, 795 \mathrm{nM}\right.$; online supplemental figure S1) and was affinity matured to yield BCY592 ( $\mathrm{K}_{\mathrm{D}}$, $41 \mathrm{nM}$; online supplemental figure S1). Chemical optimization of BCY592 led to discovery of BCY7965 with a $\mathrm{K}_{\mathrm{D}}$ of $4 \mathrm{nM}$, which represents a 200-fold increase in potency over the original phage library hit, BCY586 (online supplemental figure S1 and figure 1B). We used X-ray crystallography to characterize the mode of binding of the Bicycle to CD137. We determined the first co-crystal structure of a chemically synthetic, low-molecular-weight Bicycle bound to $\mathrm{CD} 137$ to a resolution of $2.01 \AA$, in this instance using BCY10916 which is a close analog of the affinity matured phage library hit BCY592 (figures 1C and 2A). We found that the Bicycle interacts with CD137 in the same region as CD137L (figure 1C). BCY10916 contacts cysteine-rich domains (CRDs) 2 and 3 of CD137 (figure 2A), with a footprint partially overlapping the binding surfaces for CD137L and utomilumab (figure 2A). ${ }^{21-23}$ This is consistent with the finding that Bicycles from this lead series inhibit the binding of both CD137L and utomilumab to CD137, but not urelumab, which binds distally at the N-terminal CRD1 region of the protein (figure 2B). ${ }^{21}$ Alignment of the Bicycle/CD137 structure with the CD137/ CD137L trimer complex suggests that a trimeric Bicycle would readily fit in the space normally occupied by the
CD137L trimer (figure 1C). As the physiological ligands of OX40 and CD137 induce signaling by clustering the receptors as homotrimeric complexes, ${ }^{23-25}$ we explored the use of multimeric Bicycles as TNFR family agonists. For this purpose, we synthesized 2, 3 and 4-arm polyethylene glycol (PEG) hinges with terminal azides and used "click" chemistry to add the CD137 Bicycles to form dimers, trimers and tetramers respectively (online supplemental table S3, S4). We found that the trimeric and tetrameric, but not monomeric or dimeric Bicycles acted as agonists in cellbased in vitro models (online supplemental figure S2A-E and figure 3A). Furthermore, a tetrameric CD137 Bicycle, BCY8947, like anti-CD137 agonist antibodies, abrogated the growth of MC38 tumors (online supplemental figure $\mathrm{S} 3 \mathrm{~A}, \mathrm{~B})$ and led to an increase in the numbers of $\mathrm{CD} 8+\mathrm{T}$ cells in the tumor (online supplemental figure S3C,D).

\section{Target-dependent TNFR agonists}

Next, we explored the possibility of directing CD137 Bicycles to tumors and at the same time investigated the potential for a tumor antigen to provide a scaffolding function to oligomerize the CD137 Bicycle presented to immune cells. This concept is illustrated graphically in figure 1D. We achieved this by chemically linking Bicycle binders to well-known highly expressed tumor cell antigens-Ephrin A2 receptor (EphA2), Nectin-4, and PD-L1-to those binding members of the TNFR family. For this purpose, we used 2, 3 and 4-arm PEG linkers to synthesize molecules with different stoichiometric ratios of tumor antigen to CD137/OX40 Bicycles and explored the influence of valency and linker length on agonism (online supplemental tables S3 and S5). Functional activity was tested in appropriate TNFR reporter cell assays, employing tumor 
A

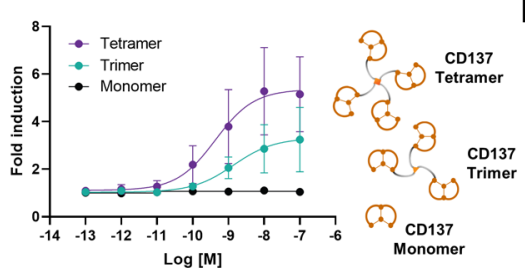

B

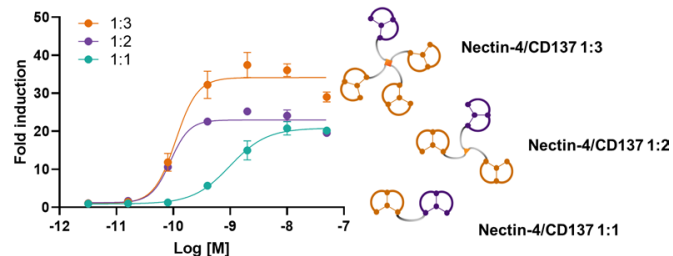

C

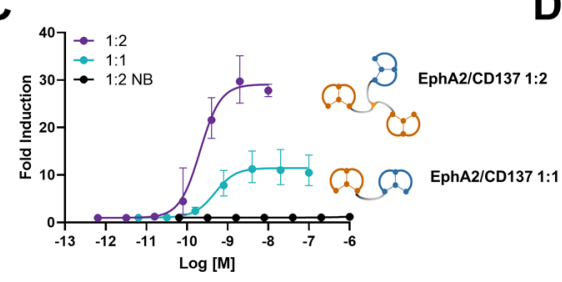

D

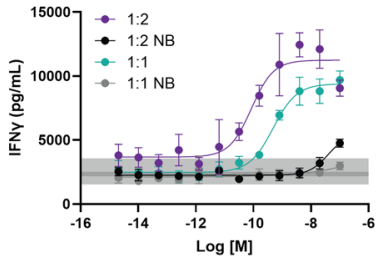

EphA2/CD137 1:2
SCa EphA2/CD1371:1

$\mathbf{F}$

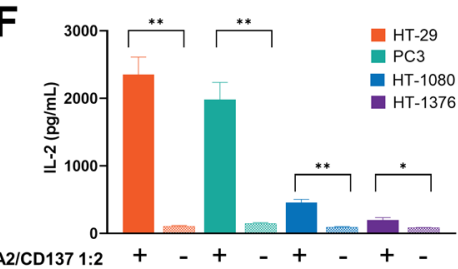

Figure 3 Synthetic CD137-binding multimeric or tumor-targeted Bicycles are agonists in vitro. (A) Jurkat cells expressing human $\mathrm{CD} 137$ coupled to a luciferase reporter gene were treated for $6 \mathrm{hr}$ with $\mathrm{CD} 137$ monomer (BCY592), trimer (BCY7750) or tetramer (BCY7751) as indicated and activity read out as reporter gene product activity. Data are mean/SD. ( $n=4$ replicates). (B) Jurkat-CD137 reporter cells were cultured with Nectin-4-expressing HT-1376 cells and treated with a Nectin-4/CD137 1:1, 1:2 or 1:3 TICAs (BCY10572, BCY11027, BCY11022, respectively) for 6 hr. Data are mean/SD. ( $n=3$ replicates). (C) Jurkat-CD137 reporter cells were cultured with EphA2-expressing A549 cells and treated with an EphA2/CD137 1:1 TICA (BCY9173), 1:2 TICA (BCY12491) or an enantiomeric non-binding control (NB) TICA (BCY13626) for $6 \mathrm{hr}$. Data are mean/SD. ( $\mathrm{n}=3$ replicates). (D) Human PBMCs (donor 228711) were cultured with mouse MC38 cells and treated with EphA2/CD137 1:1 TICA (BCY10575), 1:2 TICA (BCY12491) and enantiomeric non-CD137 binding (NB) 1:1 (BCY12759) and 1:2 (BCY12762) TICA . IFNY in the culture supernatants was measured after $48 \mathrm{hr}$. The gray bar indicates untreated control levels. Data are means and SD of 3 technical replicates. (E) and $(F)$ human PBMCs from a single donor were cultured with human cell lines treated with $3 \mathrm{nM}$ EphA2/CD137 1:2 TICAs (BCY12491). Supernatant IFN $N_{Y}$ and IL-2 were measured after $48 \mathrm{hr}$. Data are mean/SD. ( $\mathrm{n}=3$ replicates). ${ }^{*} \mathrm{P}<0.05$, ${ }^{* *} \mathrm{p}<0.01$ based on Student's t-test paired by PBMC donor.

cell lines as sources of tumor antigen in trans (figure 3B,C and online supplemental figure $\mathrm{S} 4$ ).

Turning first to the Nectin-4/CD137 constructs, we found that a simple construct comprizing one Nectin-4 binding Bicycle chemically linked to one CD137 binding Bicycle (1:1 format) was a potent agonist in a model system of CD137 reporter cells cocultured with Nectin4-expressing tumor cell lines (figure $3 \mathrm{~B}$ and online supplemental figure S4A,B). The activity was completely dependent on binding to both CD137 and Nectin-4. When a non-binding analog of the CD137 Bicycle having the same peptide sequence but constructed entirely of D-amino acids was incorporated activity was lost (online supplemental figure S4A); furthermore, PC3 cells that do not express Nectin-4 did not support CD137 agonism in the coculture reporter assay (online supplemental figure S4B). Constructs bearing two CD137 binding Bicycles (1:2 format) proved even more potent, but there was no significant improvement with a third CD137 binding Bicycle (1:3 format) (figure 3B).

We then extended the observations to analogous constructs with other tumor antigens and TNFR family receptors. Thus, the EphA2/CD137 constructs proved to be potent agonists, again with the 1:2 format more potent than the 1:1 (figure 3C). As expected, the activity was independent of the cell line presenting the target antigen (online supplemental figure S4D). The EphA2/CD137 1:2 construct exhibited a similar potency and maximal activity in the reporter cell assay as an anti-CD137 agonist antibody (online supplemental figure S4E). We similarly generated agonists from PD-L1/CD137 and Nectin-4/ OX40 target/receptor combinations (online supplemental figure $\mathrm{S} 4 \mathrm{C}, \mathrm{F})$. In the latter case, the exquisite reliance on target antigen was further demonstrated by comparing activity with Nectin-4-transfected 4T1 cells and non-expressing parental 4T1 cells (online supplemental figure S4F). Taken together, these data highlight that synthetic agonists can be readily generated by assembly of Bicycle modules, and we have termed these TICAs. Given the significant overexpression of EphA2 in lung, pancreatic and other cancers with high unmet medical need, ${ }^{1618}$ and the important role of CD137 as a costimulatory receptor with high potential in immuno-oncology, we prioritized EphA2/CD137 TICAs for further evaluation.

While the EphA2 binding Bicycles described here bind to both human and mouse EphA2, those binding 
to human CD137 do not bind to the mouse homolog (figure 1). Hence, to evaluate the behavior of EphA2/ CD137 TICAs, we used human PBMCs for in vitro studies and $\mathrm{C} 57 / \mathrm{Bl} 6$ mice transgenic for the human CD137 extracellular domain (huCD137) for in vivo studies. Human PBMCs were cultured with EphA2-expressing mouse MC38 tumor cells and then treated with antiCD3 antibodies. The generation of IFN $\gamma$ increased in the presence of EphA2/CD137 TICAs in a dose-dependent manner (figure 3D). The 1:2 format TICA (BCY12491) proved more potent than the 1:1 TICA (figure 3D), a finding consistent across PBMC donors (online supplemental figure S4G). BCY12491 was, therefore, selected for further study and shown to be potently and specifically bioactive across a range of EphA2 expressing cell lines (online supplemental figure S5). The medicinal chemistry optimization of TICAs that led to the discovery of BCY12491 will be described in detail in a separate report.

\section{EphA2/CD137-directed tumor immunity}

BCY12491 exhibited a pharmacokinetic (PK) profile in mice typical of renally eliminated hydrophilic small molecules with a terminal plasma half-life of approximately $2 \mathrm{hr}$ when dosed intraperitoneally (figure 4A). This property of BCY12491, coupled with the small size and rapid tissue penetration of this novel class of molecules, ${ }^{17}$ allowed us to test whether efficacy could be achieved with intermittent dosing where plasma drug concentrations fall to very low levels between doses, indeed well below the EC50 in the human PBMC-mouse MC38 cell coculture assays $(\sim 0.06 \mathrm{nM}$, figure 3D). We treated huCD137 transgenic C57Bl/6 mice bearing subcutaneous MC38 tumors with BCY12491 at two doses ( 5 and $15 \mathrm{mg} / \mathrm{kg}$ intraperitoneal) each with two regimens (daily qd or every third day q3d). BCY12491 was efficacious, reducing tumor growth and causing complete regressions (CRs) in every group with the rate of CRs ranging from $2 / 6(5 \mathrm{mg} / \mathrm{kg} \mathrm{q} 3 \mathrm{~d})$ to $6 / 6$ (15 mg/kg qd) (figure 4B,C). Furthermore, the CR mice
A
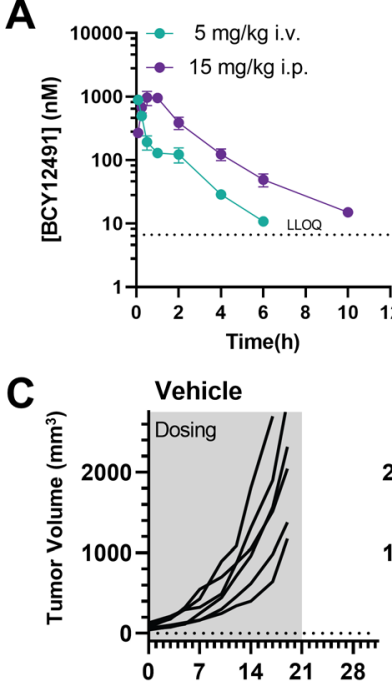

D

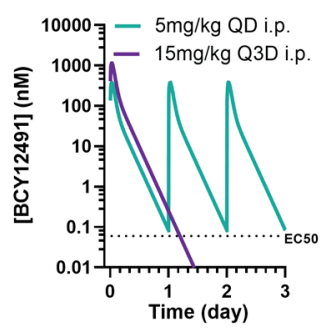

\begin{tabular}{lcc}
\hline $\begin{array}{l}\text { PK } \\
\text { Parameters }\end{array}$ & IV & IP \\
\hline Dose & 5.0 & 15 \\
\hline Vss (L/kg) & 1.5 & - \\
\hline $\mathrm{CL}$ & 17 & - \\
(mL/min/kg) & 17 & \\
\hline $\begin{array}{l}\text { Terminal t } 1 / 2 \\
\text { (h) }\end{array}$ & 1.1 & 2.0 \\
\hline & & \\
\hline
\end{tabular}

B

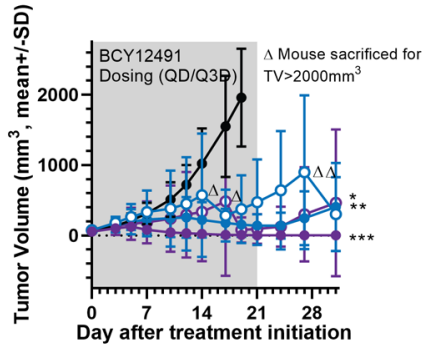

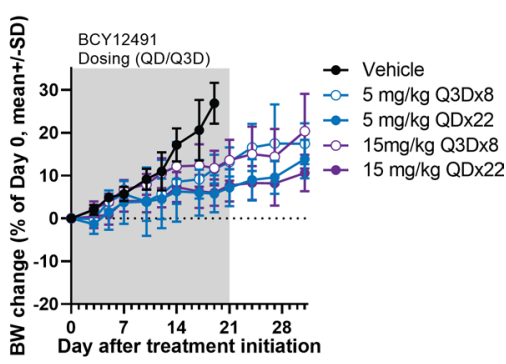
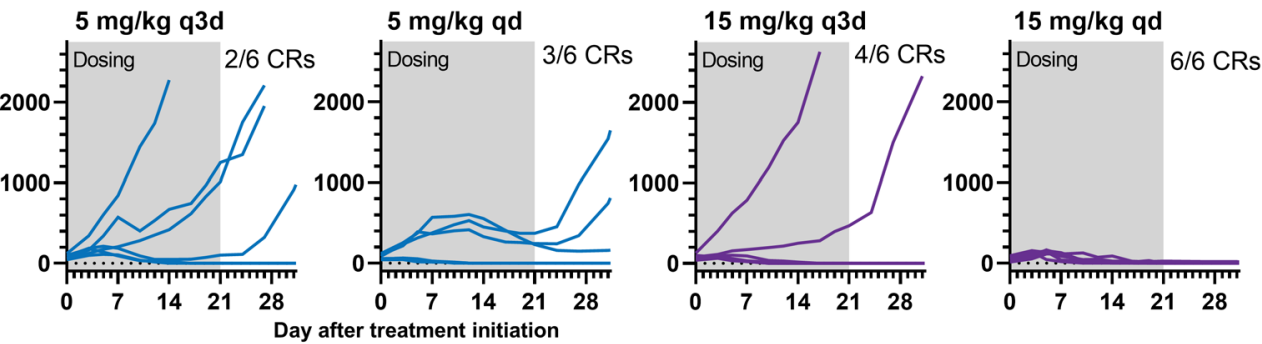

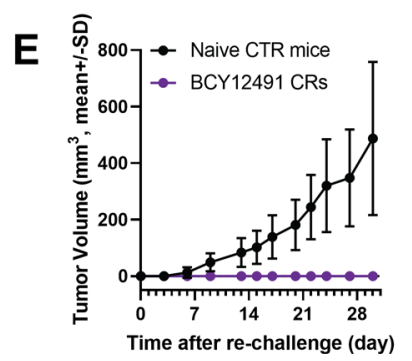

Figure 4 TICAs cause tumor regression and complete responses in vivo without continuous drug exposure in the periphery. (A) Plasma concentration of EphA2/CD137 1:2 TICA (BCY12491) after $5 \mathrm{mg} / \mathrm{kg}$ intravenous and $15 \mathrm{mg} / \mathrm{kg}$ intraperitoneal dosing in naïve CD-1 mice. Table insert shows the pharmacokinetic parameters of BCY12491 after $5 \mathrm{mg} / \mathrm{kg}$ intravenous or $15 \mathrm{mg} / \mathrm{kg}$ intraperitoneal dosing in naïve CD-1 mice. (B) MC38 tumor growth and body weight change with intermittent or daily intraperitoneal dosing of $B C Y 12491$ ( $n=6 /$ cohort; ${ }^{*} p<0.05,{ }^{* \star} p<0.01,{ }^{* \star *} p<0.001$ mixed-effects analysis days $\left.0-19\right)$. (C) Tumor growth curves for individual animals by treatment group from the experiment shown in panel B. Number of complete responders (CRs) are indicated in the figure. Gray highlighting indicates the treatment administration window. (D) Simulated plasma concentration-time profile of BCY12491 dosed QD and Q3D at 5 and $15 \mathrm{mg} / \mathrm{kg}$, respectively. The dashed line indicates the average half maximal effect concentration for BCY12491 for IFN $\gamma$ secretion from human PBMCs cultured with mouse MC38 cells from two donors (figure 2D and extended data figure $5 \mathrm{~F}$ ). (E) Five mice that had complete regressions of MC38 tumors in response to BCY12491 treatment (panel B) were rechallenged with MC38 tumor cell implantation. Tumor growth was monitored until day 31. Matched naïve huCD137 C57BL/6 mice served as a control group. $n=5 /$ cohort. 

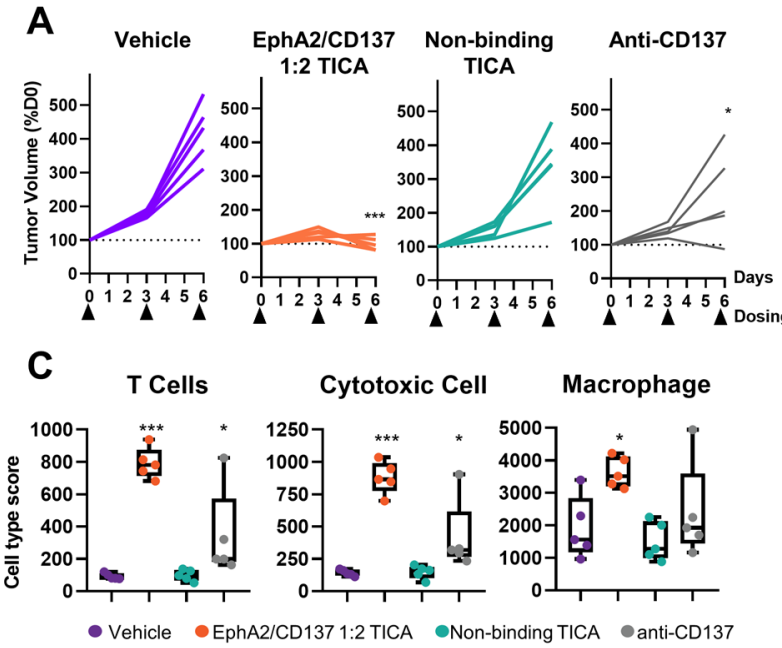

D

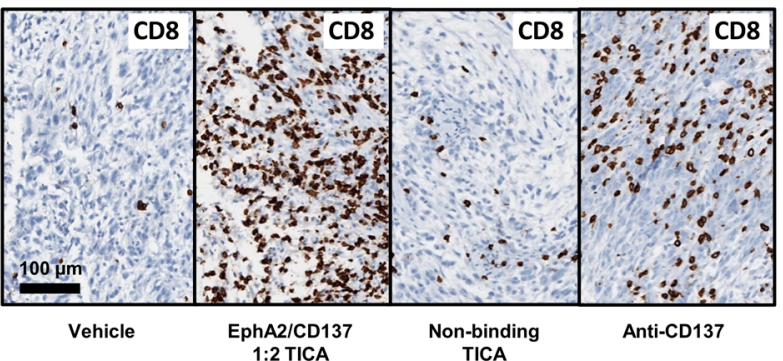

B

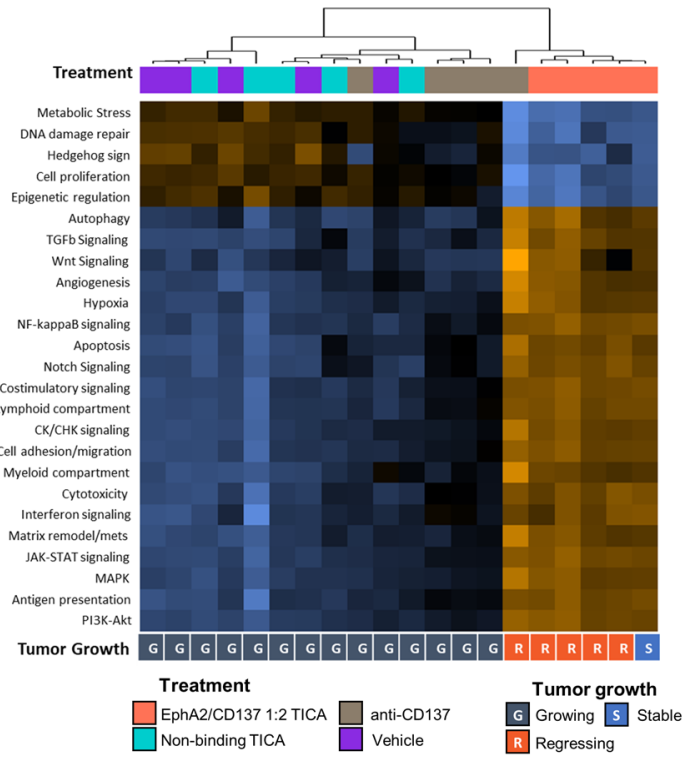

$E$

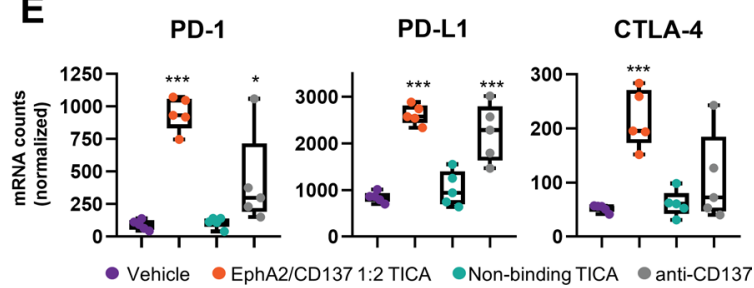

Figure 5 TICAs modulate the tumor immune microenvironment and drive T cell infiltration. (A) MC38 tumor bearing mice were treated with vehicle, $15 \mathrm{mg} / \mathrm{kg}$ EphA2/CD137 1:2 TICA (BCY12491), an enantiomeric non-binding control TICA (BCY13626) q3d intravenously or $2 \mathrm{mg} / \mathrm{kg}$ anti-CD137 agonist antibody (urelumab analog) q3d intraperitoneally individual tumor volumes normalized to tumor volume on the day of treatment initiation are shown grouped by treatment. ${ }^{*} \mathrm{P}<0.05,{ }^{* * *} \mathrm{p}<0.001$ one-way ANOVA on day 6 comparing to vehicle. (B) Nanostring analysis of tumors show the immunomodulatory effect of BCY12491 and anti-CD137 agonist antibody on the 25 functional pathways including stimulation of NF-kB signaling, costimulatory signaling, cytokine/chemokine signaling, cytotoxicity and interferon signaling among others. Orange indicates high pathway scores; blue indicates low scores. Scores are displayed on the same scale via a Z-transformation. (C) Nanostring analysis of tumors show the effect of BCY12491 and anti-CD137 agonist antibody on the T cell (probe set: Cd3d, Cd3e, Cd3g, CD6, SH2D1A and Trat1), cytotoxic cell (probe set: Ctsw, Gzma, Gzmb, Klrb1, Klrd1, Klrk1, Nkg7 and Prf1) and macrophage (probe set: CD163, CD68, CD84 and Ms4a4a) content. (D) Representative images of tissue sections from tumors treated with vehicle, $15 \mathrm{mg} / \mathrm{kg}$ BCY12491, BCY13626 or $2 \mathrm{mg} / \mathrm{kg}$ anti-CD137 agonist antibody Q3D and stained for mouse CD8 are shown. (E) Nanostring analysis of tumors show the effect of BCY12491 and anti-CD137 agonist antibody on the checkpoint inhibitor Pdcd1 (protein PD-1), Cd274 (protein PD-L1) and CTLA4 (protein CTLA-4) transcription. (C, E) ${ }^{*} P<0.05,{ }^{* \star *} \mathrm{p}<0.001$, one-way ANOVA with Dunnett's post test. ANOVA, analysis of variance; PD-L1, programmed death ligand 1; TICAs, tumor-targeted immune cell agonists.

proved resistant to rechallenge with MC38 tumor cell implantation (figure 4E).

Mechanistically, and consistent with expectations, we found that antitumor efficacy was dependent on CD8+ T cells (online supplemental figure S6). It did not appear to be dependent on NK cells although we do not know whether this would also be true in humans where NK cells express CD137 on activation and can play a key role in antitumor immunity. ${ }^{26}$ We conducted a further study with tumor-bearing animals euthanized after 6 days of treatment with BCY12491 or an anti-CD137 agonist antibody (a commercially available urelumab analog), followed by Nanostring mRNA profiling of the tumors. At that time, the tumors were either growing rapidly or beginning to regress (figure 5A). The mRNA profiling results revealed a profound reprogramming of the tumor immune microenvironment by BCY12491 and to lesser extent by anti-CD137 agonist antibody (figure 5B). Strikingly, BCY12491 caused highly significant increases in PD-L1, programmed cell death 1 and CTLA-4 mRNA levels (figure 5E), consistent with immune pressure on the tumor and the activation of a $\mathrm{T}$ cell response. These findings suggest a potential combination benefit with checkpoint therapy. Activation of $\mathrm{T}$ cell immunity was reflected in dramatic increases in the composite $\mathrm{T}$ cell and cytotoxic cell scores (figure 5C). There was also a significant increase in the macrophage cell score suggestive of a wider reprogramming beyond the $\mathrm{T}$ cell compartment (figure 5C). Findings in the anti-CD137 agonist antibodytreated mice were trending toward similar transcriptional patterns than findings after BCY12491 treatment but were less profound in general. Finally, histological analysis of 
tumors revealed a striking and pervasive $\mathrm{T}$ cell infiltration of tumors treated with BCY12941 that was completely absent in tumors harvested from control animals and notably increased in tumors harvested from anti-CD137 agonist antibody-treated animals (figure 5D).

\section{DISCUSSION/CONCLUSION}

We have demonstrated that Bicycles can be used as building blocks to create synthetic immune agonists and to direct them to tumors. An exemplar EphA2/CD137 1:2 TICA was shown to enable elimination of tumors in mice, leading to complete responders which resist further tumor challenge (Graphical abstract). This indicates the development of immunological memory in these mice and is consistent with the observations that CD137 agonism promotes the survival of activated $\mathrm{T}$ cells and the generation of memory T cells. ${ }^{27} 28$

We noted with considerable interest that the TICAs were effective despite intermittent dosing and that the 5 $\mathrm{mg} / \mathrm{kg} \mathrm{qd}$ and $15 \mathrm{mg} / \mathrm{kg} \mathrm{q} 3 \mathrm{~d}$ groups had similar average tumor growth trajectories and CR rates (figure 4B). Therefore, we modeled the plasma BCY12491 drug concentrations over 3 days in these two groups using the PK data collected in naive CD1 mice (figure 4D). In the $15 \mathrm{mg} / \mathrm{kg}$ q3d intraperitoneal group, the plasma drug concentrations are predicted to fall to extremely low levels between doses, whereas the daily dosing regimen maintains drug levels above the in vitro functional assay EC50 throughout treatment. These data show that it is not necessary to achieve continuous plasma exposure with these TICA molecules for robust anti-tumor efficacy. Although the comparisons to anti-CD137 agonist antibodies in this work are limited, we did not find categorical differences between the activity profiles of anti-CD137 agonist antibodies and Bicycle multimers or TICAs. The exception is the obligate dependency on tumor antigen for the TICAs. However, it is important to appreciate that the anti-CD137 agonist antibody urelumab failed in clinical development because of dose-limiting and unmanageable toxicity, not because it was dosed to full target engagement and found to be an ineffective treatment for cancer. ${ }^{32}$ Therefore tumor-localized activity and the ability to control peripheral exposure, both essential features of TICAs that are in stark contrast with anti-CD137 agonist antibodies, offer potential for an effective and useable medicine. We found a similar profile for a Nectin-4/CD137 TICA (unpublished data), suggesting that this is a general feature of the drug class which holds the promise of a practical dosing schedule for patients, especially for combination of TICAs with other therapeutic agents.

There are many questions remaining as this class of compounds progresses into clinical testing. What is the precise mechanism driving antitumor efficacy and how does this instruct optimal combination with other therapeutic agents? Our initial profiling points to T cell activation, as expected, but deeper profiling across time and using higher dimension methods including imaging will be required to fully understand the mechanistic basis for efficacy and contrast to other agents. We suspect that intermittent exposure leads to intermittent stimulation thus reducing activation-induced $\mathrm{T}$ cell death and prolonging the cytolytic capacity as exemplified recently by others. ${ }^{30}$ If true, such an improved quality of the immune response might afford higher response rates for any given amount of tumor mutational burden manifesting as benefit to patients additive to that of $\mathrm{T}$ cell checkpoint therapies. ${ }^{31} 32$ Studies to address the relationships between Bicycle drug plasma PK, blood and tumor receptor occupancy, biological activity and tumor growth inhibition are underway. In addition, the relationship between tumor antigen expression pattern and efficacy is of interest. In other words, does the priming of the antitumor response enabled by target-positive tumor cells enable clearance of target-negative cells? This is important as human tumors are typically heterogeneous with respect to expression of surface antigens. Finally, and of critical importance, is whether the efficacy seen in mice translates into humans with an acceptable therapeutic index. In summary, our work appears to open a new and tractable path to testing a novel class of therapeutic TNFR superfamily agonists in humans for the treatment of cancer.

Acknowledgements Sarah Gattineri for editorial assistance; Sir Gregory Winter for critical review of the manuscript. We would like to thank Charles River Laboratories for support with protein structural studies, Wuxi Apptec for support with chemistry, DMPK, in vitro and in vivo pharmacology studies.

Contributors PU, JL, KH, SB, RD, EH, MK, JKu, JKr, RL, JM, GM, ER, KVR, TS, HH, LC, KM, PB and NK designed experiments; MK, JKu, JKr, RL, JM, ER, KVR, GM and FY performed experiments; PU, JL, RD, EH, MK, JKu, JKr, RL, JM, GM, ER, TS and FY analyzed data; PU, JL, RD, KH, SB, EH, JKu, JKr, ER, LC, KM, PB and NK wrote the paper; PU, JL, KH, EH, GM, HH, LC, KM, PB and NK supervised research; KM and NK conceived of the project.

Funding This study was funded by Bicycle Therapeutics.

Competing interests All authors were full time employees of Bicycle Therapeutics at the time that the work was conducted and some own stock or stock options in Bicycle Therapeutics. PU, KM, NK and GM are named inventors on patent applications relating to compounds described in this work.

Patient consent for publication Not required.

Provenance and peer review Not commissioned; externally peer reviewed.

Data availability statement All data are available in the main text or the online supplemental materials. Crystallographic structures are available from the PDB under accession code 6Y8K.

Supplemental material This content has been supplied by the author(s). It has not been vetted by BMJ Publishing Group Limited (BMJ) and may not have been peer-reviewed. Any opinions or recommendations discussed are solely those of the author(s) and are not endorsed by BMJ. BMJ disclaims all liability and responsibility arising from any reliance placed on the content. Where the content includes any translated material, BMJ does not warrant the accuracy and reliability of the translations (including but not limited to local regulations, clinical guidelines, terminology, drug names and drug dosages), and is not responsible for any error and/or omissions arising from translation and adaptation or otherwise.

Open access This is an open access article distributed in accordance with the Creative Commons Attribution Non Commercial (CC BY-NC 4.0) license, which permits others to distribute, remix, adapt, build upon this work non-commercially, and license their derivative works on different terms, provided the original work is properly cited, appropriate credit is given, any changes made indicated, and the use is non-commercial. See http://creativecommons.org/licenses/by-nc/4.0/.

ORCID iD

Nicholas Keen http://orcid.org/0000-0003-0741-3716 


\section{REFERENCES}

1 Moran AE, Kovacsovics-Bankowski M, Weinberg AD. The TNFRs OX40, 4-1BB, and CD40 as targets for cancer immunotherapy. Curr Opin Immunol 2013;25:230-7.

2 Segal NH, He AR, Doi T, et al. Phase I Study of Single-Agent Utomilumab (PF-05082566), a 4-1BB/CD137 Agonist, in Patients with Advanced Cancer. Clin Cancer Res 2018;24:1816-23.

3 Segal NH, Logan TF, Hodi FS, et al. Results from an integrated safety analysis of Urelumab, an agonist Anti-CD137 monoclonal antibody. Clin Cancer Res 2017;23:1929-36.

4 Garber K. Immune agonist antibodies face critical test. Nat Rev Drug Discov 2020;19:3-5.

5 Mak IW, Evaniew N, Ghert M. Lost in translation: animal models and clinical trials in cancer treatment. Am J Trans/ Res 2014;6:114-8.

6 Schmidt MM, Wittrup KD. A modeling analysis of the effects of molecular size and binding affinity on tumor targeting. Mol Cancer Ther 2009;8:2861-71.

7 Attanasio J, Wherry EJ. Costimulatory and Coinhibitory receptor pathways in infectious disease. Immunity 2016;44:1052-68.

8 Wijewarnasuriya D, Bebernitz C, Lopez AV, et al. Excessive costimulation leads to dysfunction of adoptively transferred $T$ cells. Cancer Immunol Res 2020;8:732-42.

9 Qi X, Li F, Wu Y, et al. Optimization of 4-1BB antibody for cancer immunotherapy by balancing agonistic strength with Fc $\gamma R$ affinity. Nat Commun 2019;10:2141.

10 Bartkowiak T, Jaiswal AR, Ager CR, et al. Activation of 4-1BB on liver myeloid cells triggers hepatitis via an Interleukin-27-Dependent pathway. Clin Cancer Res 2018;24:1138-51.

11 Eskiocak U, Guzman W, Wolf B, et al. Differentiated agonistic antibody targeting CD137 eradicates large tumors without hepatotoxicity. JCI Insight 2020;5:e133647.

12 Compte M, Harwood SL, Muñoz IG, et al. A tumor-targeted trimeric 4-1BB-agonistic antibody induces potent anti-tumor immunity without systemic toxicity. Nat Commun 2018;9:4809.

13 Claus C, Ferrara C, Xu W, et al. Tumor-Targeted 4-1BB agonists for combination with $\mathrm{T}$ cell bispecific antibodies as off-the-shelf therapy. Sci Trans/ Med 2019;11:eaav5989.

14 Hinner MJ, Aiba RSB, Jaquin TJ, et al. Tumor-Localized costimulatory T-cell engagement by the 4-1BB/HER2 bispecific Antibody-Anticalin fusion PRS-343. Clin Cancer Res 2019;25:5878-89.

15 Heinis C, Rutherford T, Freund S, et al. Phage-Encoded combinatorial chemical libraries based on bicyclic peptides. Nat Chem Biol 2009;5:502-7.

16 Mudd GE, Brown A, Chen L, et al. Identification and optimization of EphA2-Selective bicycles for the delivery of cytotoxic Payloads. J Med Chem 2020;63:4107-16.
17 Eder M, Pavan S, Bauder-Wüst U, et al. Bicyclic peptides as a new modality for imaging and targeting of proteins overexpressed by tumors. Cancer Res 2019;79:841-52.

18 Bennett G, Brown A, Mudd G, et al. MMAE Delivery Using the Bicycle Toxin Conjugate BT5528. Mol Cancer Ther 2020;19:1385-94.

19 Teufel DP, Bennett G, Harrison H, et al. Stable and long-lasting, novel bicyclic peptide plasma kallikrein inhibitors for the treatment of diabetic macular edema. J Med Chem 2018;61:2823-36.

20 Smith GP. Filamentous fusion phage: novel expression vectors that display cloned antigens on the virion surface. Science 1985;228:1315-7.

21 Chin SM, Kimberlin CR, Roe-Zurz Z, et al. Structure of the 4-1BB/4$1 \mathrm{BBL}$ complex and distinct binding and functional properties of utomilumab and urelumab. Nat Commun 2018;9:4679.

22 Bitra A, Doukov T, Wang J, et al. Crystal structure of murine 4-1BB and its interaction with 4-1BBL support a role for galectin-9 in 4-1BB signaling. J Biol Chem 2018;293:1317-29.

$23 \mathrm{Li} \mathrm{Y,} \mathrm{Tan} \mathrm{S,} \mathrm{Zhang} \mathrm{C,} \mathrm{et} \mathrm{al.} \mathrm{Limited} \mathrm{cross-linking} \mathrm{of} \mathrm{4-1BB} \mathrm{by} \mathrm{4-1BB}$ ligand and the agonist monoclonal antibody Utomilumab. Cell Rep 2018;25:909-20.

24 Zapata JM, Perez-Chacon G, Carr-Baena P, et al. Cd137 (4-1BB) signalosome: complexity is a matter of TRAFs. Front Immunol 2018;9:2618.

25 Compaan DM, Hymowitz SG. The crystal structure of the costimulatory OX40-OX40L complex. Structure 2006;14:1321-30.

26 Shimasaki N, Jain A, Campana D. Nk cells for cancer immunotherapy. Nat Rev Drug Discov 2020;19:200-18.

27 Weigelin B, Bolaños E, Teijeira A, et al. Focusing and sustaining the antitumor CTL effector killer response by agonist anti-CD137 mAb. Proc Natl Acad Sci U S A 2015;112:7551-6.

28 Bertram EM, Lau P, Watts TH. Temporal segregation of 4-1BB versus CD28-mediated costimulation: 4-1BB ligand influences $T$ cell numbers late in the primary response and regulates the size of the T cell memory response following influenza infection. J Immunol 2002;168:3777-85

29 Sznol M, Hodi FS, Margolin K, et al. Phase I study of BMS-663513, a fully human anti-CD137 agonist monoclonal antibody, in patients (PTS) with advanced cancer (Ca). J Clin Oncol 2008;26:(15_suppl):3007

30 Chiu D, Tavaré R, Haber L, et al. A PSMA-targeting CD3 bispecific antibody induces antitumor responses that are enhanced by 4-1BB costimulation. Cancer Immunol Res 2020;8:596-608.

31 Ott PA, Bang Y-J, Piha-Paul SA, et al. T-Cell-Inflamed gene-expression profile, programmed death ligand 1 expression, and tumor mutational burden predict efficacy in patients treated with pembrolizumab across 20 cancers: KEYNOTE-028. J Clin Oncol 2019;37:318-27.

32 Passaro A, Stenzinger A, Peters S. Tumor mutational burden as a pan-cancer biomarker for immunotherapy: the limits and potential for convergence. Cancer Cell 2020;38:624-5. 